\title{
MULTI-CLASS TRAFFIC MODELS ON ROAD NETWORKS*
}

\author{
M. HERTY ${ }^{\dagger}$, C. KIRCHNER ${ }^{\ddagger}$, AND S. MOUTARI ${ }^{\S}$
}

Abstract. We consider a multi-class (resp. source-destination) model for traffic flow as introduced in [10]. We propose a reformulation and discuss the extension of this model to road networks by proposing criteria for solving Riemann problems at a road intersection. We present some numerical simulations for the derived conditions.

Key words. Traffic flow, networks, multi-class models.

AMS subject classifications. 35L, 35L65

\section{Introduction}

Traffic flow on a single road has been studied throughout the last 50 years and a first macroscopic model is due to Lighthill and Whitham [23] and Richards [25] (LWR-model). Various modifications and extensions to this basic model have been studied and discussed, cf. [24, 1, 11, 8, 2]. Today, fluid dynamic models for traffic flow are appropriate to describe traffic phenomena as for example congestion and stop-and-go waves $[17,12,19]$. Recently, there has been intensive research on traffic flow for road networks in the mathematical $[5,9,16,14,10,15]$ as well as in the engineering community [21, 22].

We are interested in multi-class traffic flow models, i.e., models containing two or more car species, e.g. cars having different destinations, cf. [10]. Such models have been proposed for example in $[3,4,27,28,26]$ and in [10]. The latter publication introduces a source-destination model based on the LWR equation for a road network and analyzes its mathematical properties [10]. We consider a reformulation of this model, which then can also be seen as a particular case of the multi-class model introduced and discussed for example in $[27,26]$, see below for the details. The reformulation of the multi-class or source-destination model of [10] allows for an alternative extension to road networks. In particular, the discussion of suitable coupling conditions for this model at road intersections has to be adapted to the reformulation and we introduce a different modeling of the coupling conditions, cf. Section 3. Similar to the approach in $[16,15,5]$, existence of solutions is granted by an analysis of the arising (half-) Riemann problems. Moreover, we identify supply and demand functions $[21,7,8]$ in this context and present some numerical examples.

The paper is organized as follows. In Section 2 we recall the multi-class or sourcedestination model, respectively, and state some analytical properties. In Section 3 we present the (half-) Riemann problems and their solution. We give numerical examples in Section 4.

\footnotetext{
${ }^{*}$ Received: March 30, 2006; accepted (in revised version): July 7, 2006. Communicted by Lorenzo Pareschi.

${ }^{\dagger}$ Fachbereich Mathematik, TU Kaiserslautern, D-67653 Kaiserslautern, Germany (herty@ mathematik.uni-kl.de).

‡Fachbereich Mathematik, TU Kaiserslautern, D-67653 Kaiserslautern, Germany (kirchner@ mathematik.uni-kl.de).

$\S$ Laboratoire J. A. Dieudonné, UMR CNRS $N^{o}$ 6621, Université de Nice-Sophia Antipolis, Parc Valrose, 06108 Nice Cedex 2, France (salissou@math.unice.fr).
} 


\section{Preliminary Discussion}

We derive the source-destination model of [10] by starting from the multi-class model of $[27,26]$. The relation between both models is given below in Remark 2.1.

A multi-class (or multi-population) model based on the LWR equation for different car classes has been introduced in $[27,26,4]$. They consider a continuum model for traffic flow with heterogeneous $m$ media. Therein, $\rho_{j}$ is the density of the $j^{\text {th }}$ class and the velocity field $v_{j}$ of the $j^{\text {th }}$ class is assumed to be a function of all densities $\left\{\rho_{j}\right\}_{j=1}^{m}$. Then, the mass conservation for the $j^{t h}$ class yields the multi-class LWR-type model:

$$
\partial_{t} \rho_{j}+\partial_{x}\left(\rho_{j} v_{j}\right)=0, j=1, \ldots, m \text {. }
$$

The velocity fields $v_{j}$ are required to satisfy additional conditions to obtain a hyperbolic system and we refer the reader to [27] and [4] for more details. A particular choice for the velocity fields is given by

$$
v_{j}\left(\left(\rho_{j}\right)_{j=1}^{m}\right)=v_{j}\left(\sum_{j=1}^{m} \rho_{j}\right)=v_{j}^{f} \cdot\left(1-\frac{\sum_{j} \rho_{j}}{\rho_{\max }}\right), j=1, \ldots, m,
$$

where $v_{j}^{f}$ is the constant class dependent free flow velocity and $\rho_{\max }$ is the maximal density for the total (car-)density

$$
\rho:=\sum_{j} \rho_{j} .
$$

In this case and for $m=1$ equations (2.1) and (2.2) obviously reduce to the standard LWR traffic flow model:

$$
\partial_{t} \rho+\partial_{x}(\rho v(\rho))=0 .
$$

Note that if $v_{j}^{f}$ is the same for all cars, i.e. car species have the same fundamental diagram $\left(\rho_{j}, \rho_{j} v(\rho)\right)$, then, the model $(2.1,2.2)$ can be interpreted as a model for a car species $j$ having properties which are independent of the dynamics of the actual class $j$. For instance, one can think of classes of cars having different destinations $d_{j}$. A model for classes of cars with different source-destination pairings has been recently introduced in [10]. We recall this model in Remark 2.1 and show its relation to the multi-class model stated above.

REMARK 2.1. In [10] a source-destination model for road networks is considered. They introduce functions $\pi^{j}, j=1, \ldots, m$ which specify the amount of car density $\rho$ going from a specific source $s_{j}$ to a destination $d_{j}$. The dynamics for the car density are governed by the LWR equation and, therefore, the following system for a single road is derived:

$$
\begin{aligned}
\partial_{t} \rho+\partial_{x}(\rho v(\rho)) & =0, \\
\partial_{t} \pi^{j}+v(\rho) \partial_{x} \pi^{j} & =0, j=1, \ldots, m, \\
\sum_{j} \pi^{j} & =1 .
\end{aligned}
$$


If we define

$$
\rho_{j}:=\pi^{j} \rho,
$$

then at least formally,

$$
\partial_{t} \rho_{j}=\pi^{j} \partial_{t} \rho+\rho \partial_{t} \pi^{j}=-\partial_{x}\left(\rho_{j} v(\rho)\right),
$$

i.e., we recover the multi-class model (2.1) for the particular velocity fields

$$
v_{j} \equiv v, j=1, \ldots, m \text {. }
$$

In [10] the extension of (2.5) to networks is performed by defining coupling conditions at road intersections. Here, and in contrast to the work of [10], we discuss the extension of the reformulation (2.1),(2.8) to networks and present numerical results. This allows for an alternative view on the coupling conditions and induces in particular the possibility to define class-specific distribution rates of cars at junctions.

Having the previous remark in mind, we consider the reformulated version of the source-destination (resp. multi-class) model. In this study, we restrict ourselves to the case $m=2$. The proofs following are limited to this case only. For a discussion of hyperbolicity and properties of the set of equations in the case $m>2$ for a single road, we refer to the publications of Zhang et. al., and in particular to [27].

We normalize the total density $\rho_{\max }=1$ and set the free flow velocities $v_{1}^{f}=v_{2}^{f}=c$, $c>0$ constant. Hence, we might allow different total free flow velocities on different parts of the road network. Finally, we obtain the following system for the evolution of a two-class model on a single road $x \in[a, b], t>0$,

$$
\frac{\partial}{\partial t}\left(\begin{array}{c}
\rho_{1} \\
\rho_{2}
\end{array}\right)+\frac{\partial}{\partial x}\left(\begin{array}{c}
\rho_{1} v(\rho) \\
\rho_{2} v(\rho)
\end{array}\right)=\left(\begin{array}{l}
0 \\
0
\end{array}\right),
$$

where $\rho=\rho_{1}+\rho_{2}$ and (cf. $(2.2)$ ),

$$
v(\rho)=v\left(\rho_{1}+\rho_{2}\right)=c\left(1-\left(\rho_{1}+\rho_{2}\right)\right) .
$$

For the discussion of suitable coupling conditions at a road intersection, we need to recall the following properties of the hyperbolic system (2.9). Let $U=\left(\rho_{1}, \rho_{2}\right)$. Then [27], the eigenvalues are

$$
\lambda_{1}(U)=c \cdot\left(1-2\left(\rho_{1}+\rho_{2}\right)\right) \leq \lambda_{2}(U)=c \cdot\left(1-\left(\rho_{1}+\rho_{2}\right)\right) .
$$

Hence, away from the vacuum $U=(0,0)$, the system is strictly hyperbolic. The eigenvectors corresponding to $\lambda_{1}$ and $\lambda_{2}$ are respectively

$$
r_{1}(U)=\left(\begin{array}{c}
\frac{\rho_{1}}{\rho_{2}} \\
1
\end{array}\right), \quad r_{2}(U)=\left(\begin{array}{c}
-1 \\
1
\end{array}\right)
$$

and the characteristic family associated with the first eigenvalue is genuinely nonlinear (GNL) whereas the second characteristic family is linearly degenerate (LD). The Riemann invariants in the sense of Lax (RI-Lax) are

$$
w(U)=\log \left(\frac{\rho_{1}}{\rho_{2}}\right) \text { and } z(U)=c \cdot\left(1-\left(\rho_{1}+\rho_{2}\right)\right)=v(U),
$$


respectively. Next, we state the elementary waves [6] of (2.9).

An arbitrary left state $U^{-}=\left(\rho_{1}^{-}, \rho_{2}^{-}\right)>0$ can be connected to a state $U^{+}$by a 1-rarefaction wave, iff $U^{+}=U(\xi)$ for $0<\xi \leq \rho_{1}^{-}$and where $\xi \rightarrow U(\xi)$ is the following parametrization of the 1-rarefaction wave curve:

$$
U(\xi)=\left(\begin{array}{c}
1 \\
\frac{\rho_{2}^{-}}{\rho_{1}^{-}}
\end{array}\right) \xi, \quad 0<\xi \leq \rho_{1}^{-} .
$$

Therefore, in the phase plane $\left(\rho_{1}, \rho_{2}\right)$, the 1-rarefaction curve is a straight line of slope $\frac{\rho_{2}^{-}}{\rho_{1}^{-}}$as depicted in Figure 2.1.

Next, we consider the 1-(Lax)-shock waves: A left state $U^{-}=\left(\rho_{1}^{-}, \rho_{2}^{-}\right)>0$ can be connected to any other state $U^{+}=\left(\rho_{1}^{+}, \rho_{2}^{+}\right)>0$ by a 1-shock wave, iff $U^{+}=U(\xi)$ for some $\xi$ where $\xi \rightarrow U(\xi)=\left(\rho_{1}(\xi), \rho_{2}(\xi)\right)$ is a parametrization of the 1 -shock wave curve:

$$
U(\xi)=\left(\begin{array}{c}
1 \\
\frac{\rho_{2}^{-}}{\rho_{1}^{-}}
\end{array}\right) \xi, \quad \rho_{1}^{-} \leq \xi \leq \frac{\rho_{1}^{-}}{\rho_{1}^{-}+\rho_{2}^{-}} .
$$

The shock speed $s_{1}$ is given by

$$
s_{1}=c\left(1-\left(\rho_{1}(\xi)+\rho_{2}(\xi)+\rho^{-}\right)\right),
$$

and for system (2.9), the 1-rarefaction and 1-shock wave curves coincide, cf. Figure 2.1. In fact, equation (2.9) is a Temple system.

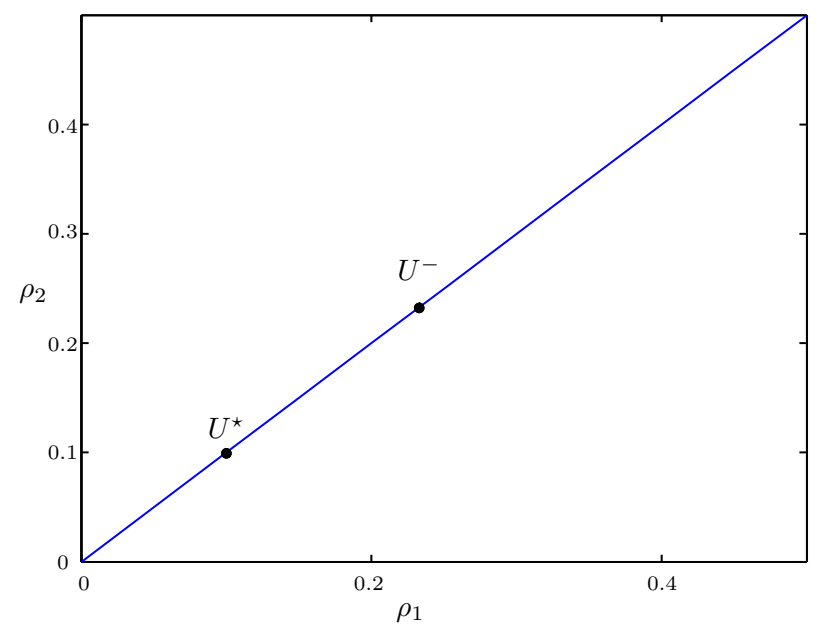

FIG. 2.1. The 1-rarefaction and 1-shock curves in the phase plane $\left(\rho_{1}, \rho_{2}\right)$.

It remains to discuss the 2-contact discontinuities. A left state $U^{-}=\left(\rho_{1}^{-}, \rho_{2}^{-}\right)$can be connected to $U^{+}=\left(\rho_{1}^{+}, \rho_{2}^{+}\right)$, iff $U^{+}=U(\xi)$ where $U(\xi)$ is given by

$$
U(\xi)=\left(\begin{array}{c}
1 \\
-1
\end{array}\right) \cdot \xi+\left(\begin{array}{c}
0 \\
\rho^{-}
\end{array}\right) \quad 0 \leq \xi \leq \rho^{-}
$$


Of course, $v\left(U^{+}\right)=v(U(\xi))=v\left(U^{-}\right)$for all $0 \leq \xi \leq \rho^{-}$and in the phase plane the 2 -contact wave curve is a straight line through $U^{-}$with slope -1 .

Now, a Riemann problem for (2.9) is a Cauchy problem for (2.9) with the piecewise constant initial data

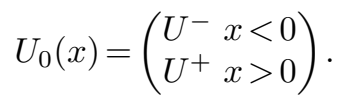

From the above discussion, we obtain that the Riemann problem with data $U^{-}$ and $U^{+}$and such that $0<\rho^{-} \leq 1$ and $0<\rho^{+} \leq 1$, always admits a weak entropy solution [20]. In general, a solution is a composition of a 1-(Lax-)shock or 1-rarefaction wave connecting $U^{-}$to $U^{*}$,

$$
U^{*}:=\frac{\rho^{+}}{\rho^{-}}\left(\begin{array}{c}
\rho_{1}^{-} \\
\rho_{2}^{-}
\end{array}\right)
$$

and a 2-contact discontinuity connecting $U^{*}$ with $U^{+}$, see Figure 2. Hence, we call initial data $U=\left(\rho_{1}, \rho_{2}\right)$ admissible, if it satisfies $0<\rho_{1}+\rho_{2} \leq 1$.

Proposition 2.1. Consider admissible initial data $U^{-}$and $U^{+}$. Then, the Riemann problem for equation (2.9) admits a unique weak entropy solution $U(x, t)$.
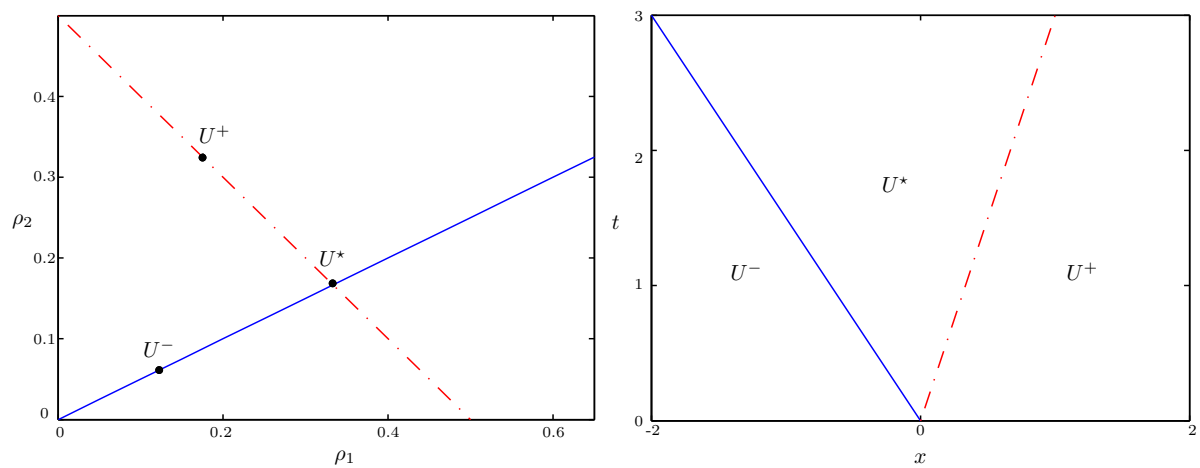

FIG. 2.2. The solution to a Riemann problem in the phase plane (right), in the (x,t) plane (left).

Before we discuss the extensions of the above model to the network, we introduce the notion of supply and demand functions, cf. [21, 7]. This will be used later to determine the range of admissible states at a junction, see below. The supply and demand functions are defined in the $(\rho, \rho v)$-plane with $\rho=\rho_{1}+\rho_{2}$ and $\rho v$ being the total flux. Recalling Remark 2.1 (and [10], respectively), it is not surprising, that the 1 - and 2-wave curves, allow for a particular simple characterization in the $(\rho, \rho v)$ plane.

Proposition 2.2. Consider an admissible left state $U^{-}=\left(\rho_{1}^{-}, \rho_{2}^{-}\right)$. Then, there exists a one-to-one correspondence between the states $U^{+}=\left(\rho_{1}^{+}, \rho_{2}^{+}\right)$, which can be connected by either a 1-rarefaction or 1-shock wave to $U^{-}$, and the points $(\eta, \eta v(\eta))$, $0<\eta \leq 1$, of the $(\rho, \rho v)$-plane, such that $\eta=\rho_{1}^{+}+\rho_{2}^{+}$holds.

Here, $v(\eta)=c(1-\eta)$ is as in (2.10). 
Proof. A state $U^{+}$can be connected by a 1 -rarefaction or 1 -shock wave to $U^{-}$, iff $U^{+}=U(\xi)$ and $U(\xi)$ is given by either (2.14) or (2.15), i.e.,

$$
U(\xi)=\left(\begin{array}{c}
1 \\
\rho_{2}^{-} / \rho_{1}^{-}
\end{array}\right) \xi, \quad 0<\xi \leq \frac{\rho_{1}^{-}}{\rho_{1}^{-}+\rho_{2}^{-}} .
$$

Therefore, $\rho(\xi)=\rho_{1}(\xi)+\rho_{2}(\xi)=\xi\left(1+\rho_{2}^{-} / \rho_{1}^{-}\right)$and satisfies $0<\eta:=\rho(\xi) \leq 1$.

The mapping $\eta \rightarrow \eta v(\eta)$ is usually referred to as a fundamental diagram. Similarly, we obtain the following assertion for the states $U^{+}$which can be connected to $U^{-}$by a wave of the second family:

Proposition 2.3. Consider an admissible left state $U^{-}=\left(\rho_{1}^{-}, \rho_{2}^{-}\right)$. Then, the set of all states $U^{+}$which can be connected to $U^{-}$by a wave of the second family is mapped to the single point $(\eta, \eta v(\eta)), \eta=\rho_{1}^{-}+\rho_{2}^{-}$, in the $(\rho, \rho v)-$ plane.

Proof. This is immediate since $U^{+}=U(\xi)$ with $U(\xi)$ given by equation (2.17) and hence satisfies $\rho(\xi)=\rho^{-}$.

As in $[15,21]$, we introduce the demand and supply functions defined in the $(\rho, \rho v)$-plane: The demand function $\eta \rightarrow d(\eta)$ and the supply function $\eta \rightarrow s(\eta)$ are the non-decreasing (see Figure 2.3) and the non-increasing (see Figure 2.4) parts of the curve $\eta \rightarrow \eta v(\eta)$ in the $(\rho, \rho v)$-plane, respectively.

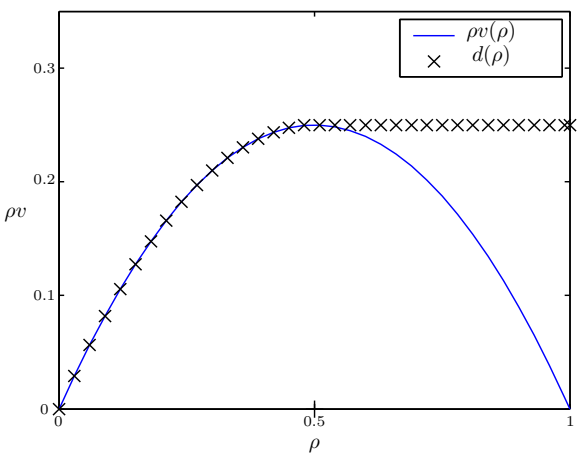

FIG. 2.3. Demand function in the $(\rho, \rho v)$

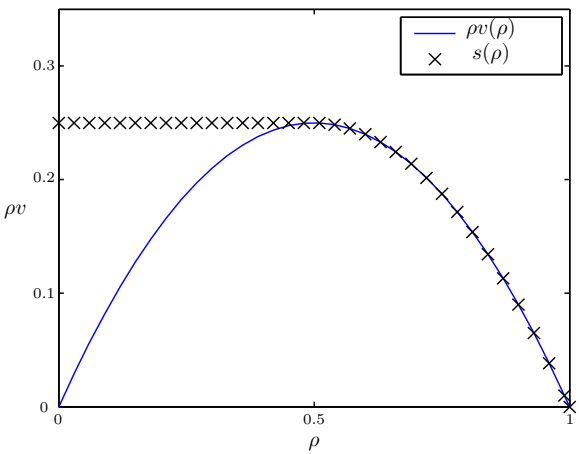

FIG. 2.4. Supply function in the $(\rho, \rho v)$ plane.

This finishes the characterization of states $U^{ \pm}$on the 1 - and $2-$ wave curves. We will reconsider the supply and demand notion when defining solutions to a network problem.

\section{Extension to a network}

As in [16], we model a road network as a finite direct graph $(\mathcal{I}, \mathcal{N})$ with $|\mathcal{I}|=\mathbf{I}$ and $|\mathcal{N}|=\mathbf{N}$. Each arc $i$ corresponds to a road and each vertex $n$ to a junction. For a fixed junction $n$, we denote by $\delta_{n}^{-}$the set of all its incoming roads whereas $\delta_{n}^{+}$is the set of all its outgoing roads. We parameterize each road $i$ by an interval $\left[a^{i}, b^{i}\right]$ with possibly $a^{i}=-\infty$ or $b^{i}=\infty$. At a fixed vertex $n$, we set $x_{n}^{k}=b^{k}$ if $k \in \delta_{n}^{-}$and $x_{n}^{k}=a^{k}$, if $k \in \delta_{n}^{+}$.

We use upper indices to index the arc and on each road $i$ we consider system (3.1) 


$$
\partial_{t}\left(\begin{array}{c}
\rho_{1}^{i} \\
\rho_{2}^{i}
\end{array}\right)+\partial_{x}\left(\left(\begin{array}{c}
\rho_{1}^{i} \\
\rho_{2}^{i}
\end{array}\right) v^{i}\left(\rho^{i}\right)\right)=0, \quad x \in\left[a^{i}, b^{i}\right], \quad t>0,
$$

where as before

$$
U^{i}:=\left(\rho_{1}^{i}, \rho_{2}^{i}\right), \quad \rho^{i}=\rho_{1}^{i}+\rho_{2}^{i}, v^{i}\left(\rho^{i}\right)=c^{i}\left(1-\rho^{i}\right),
$$

and for notational convenience $F^{i}(U)=\left(\rho_{1}, \rho_{2}\right) v^{i}(U)$. Note, that we explicitly allow for different velocity profiles on different roads (modeled by arc dependent constants $\left.c^{i}\right)$.

As in [16], we call a set of functions $\left\{U^{i}\right\}_{i \in \mathcal{I}}$ a weak solution of system (3.1), if it satisfies

$$
\sum_{i \in \mathcal{I}} \int_{0}^{\infty} \int_{a_{i}}^{b_{i}} U^{i} \cdot \partial_{t} \phi^{i}+F^{i}\left(U^{i}\right) \cdot \partial_{x} \phi^{i} d x d t=0
$$

for all families of test functions $\left\{\phi^{i}\right\}_{i \in \mathcal{I}}$ where each function $\phi^{i}: \mathbb{R}^{+} \times\left[a^{i}, b^{i}\right] \rightarrow \mathbb{R}^{2}$ is compactly supported in $(0, \infty) \times\left[a^{i}, b^{i}\right]$ and is smooth across a vertex $n: \phi^{k}\left(x_{n}^{k}, t\right)=$ $\phi^{j}\left(x_{n}^{j}, t\right)$ for $k \in \delta_{n}^{-}$and $j \in \delta_{n}^{+}$.

From (3.3) we derive the Rankine-Hugoniot conditions (3.4) as coupling conditions at a vertex $n$ :

$$
\sum_{k \in \delta_{n}^{-}} \rho_{i}^{k} v^{k}\left(\rho^{k}\right)\left(x_{n}^{k}, t\right)=\sum_{j \in \delta_{n}^{+}} \rho_{i}^{j} v^{j}\left(\rho^{j}\right)\left(x_{n}^{j}, t\right), t>0, i=1,2 .
$$

The conditions (3.4) state the conservation of each class of cars through the intersection. Frequently, we will call $\rho_{i}^{k} v^{k}\left(\rho^{k}\right)$, the $i$-th moment. Depending on the degree of the vertex, additional conditions have to be imposed to obtain a unique solution.

A major step in the construction of a solution is the consideration of half-Riemann problems at a junction. A half-Riemann problem at a vertex $n \in \mathcal{N}$ is obtained by considering $\left|\delta_{n}\right|$ Riemann problems, one on each arc $j \in \delta_{n}$, and each arc considered as extended to $(-\infty, \infty)$. Depending whether the arc is in- or outgoing to the vertex, either the left or the right data is given by the initial data $U_{k, 0}$, i.e., we consider the Riemann problems:

$$
\begin{aligned}
\frac{\partial}{\partial t} U^{k}+\frac{\partial}{\partial x} F^{k}\left(U^{k}\right) & =0, \\
U^{k}(x, 0) & = \begin{cases}U^{-} & x<x_{n}^{k}, \\
U^{+} & x>x_{n}^{k},\end{cases}
\end{aligned}
$$

where for $k \in \delta_{n}^{-}$, only the data $U^{-}$is given by the initial data, and for $k \in \delta_{n}^{+}$only the data $U^{+}$is given by the initial data $U_{k, 0}=U^{k}(x, 0)$.

Below, in proposition 3.1 and 3.2 we identify possible states $U^{+}$and $U^{-}$, respectively, such that the waves in the solution to (3.5) have either non-positive $\left(k \in \delta_{n}^{-}\right)$ or non-negative speed $\left(k \in \delta_{n}^{+}\right)$. It turns out that the simplest way to describe the admissible states $U^{ \pm}$employs the notion of supply and demand function, cf. the discussion in the previous section. In the sequel, we drop the index $k$ whenever the intention is clear. 
3.1. Admissible Riemann data for in- and outgoing roads. First, we consider the case of $k \in \delta_{n}^{-}$, i.e., an ingoing road to a vertex. Here the initial data $U^{-}$ for (3.5) is given $\left(U^{-}=U_{k, 0}\right)$ and we determine all states $U^{+}$, such that the solution $U(x, t)$ to $(3.5)$ is either a constant or contains waves of negative speed, only. We call such states $U^{+}$admissible for the incoming road. For the same reasoning as in [16], we excluded stationary shocks. Since the wave speed of waves of the second family is $v(U) \geq 0$, the only states $U^{+}$which can be connected to $U^{-}$have to be on the 1 -wave curve through $U^{-}$. More precisely, we have:

Proposition 3.1. Let $U^{-}=\left(\rho_{1}^{-}, \rho_{2}^{-}\right)$be an admissible initial value (i.e. $U^{-} \neq 0$, $\left.\rho_{1}^{-}+\rho_{2}^{-} \leq 1\right)$ on an incoming road $k$. Then, for any given flux $q \in \mathbb{R}$, such that

$$
0<q \leq d\left(\rho^{-}\right)
$$

there exists exactly one state $U^{+}$which is admissible for the incoming road and satisfies $\rho^{+} v\left(\rho^{+}\right)=q$.

Proof. Since we neglect stationary shocks, we set $U^{+} \equiv U^{-}$in the case $q=d\left(\rho^{-}\right)$. Assume now $\eta<d\left(\rho^{-}\right)$. Due to Proposition 2.2, we find for each state $U^{+}$a uniquely determined state $(\bar{\rho}, \bar{\rho} \bar{v})$ in the $(\rho, \rho v)$-plane such that $\bar{\rho}=\rho_{1}^{+}+\rho_{2}^{+}$. Next, we prove that also the wave speed of a 1-wave can be obtained by considering the data in the $(\rho, \rho v)$-plane.

If $U^{+}=\left(\rho_{1}^{+}, \rho_{2}^{+}\right)$is connected to $U^{-}$by a 1 -rarefaction wave, the corresponding wave speed is given by

$$
\lambda_{1}(U)=c\left(1-2\left(\rho_{1}+\rho_{2}\right)\right)=\partial_{\eta}(\eta v(\eta))_{\eta=\rho_{1}+\rho_{2}} .
$$

If $U^{+}$is connected to $U^{-}$by a 1 -shock, the corresponding shock speed is given by equation (2.16), i.e.,

$$
s_{1}=c\left(1-\rho_{1}^{+}-\rho_{2}^{+}-\rho_{1}^{-}-\rho_{2}^{-}\right)=\frac{(\eta v(\eta))_{\eta=\rho^{+}}-(\eta v(\eta))_{\eta=\rho^{-}}}{\rho^{+}-\rho^{-}}=\frac{[\eta v(\eta)]}{[\eta]} .
$$

Therefore, the wave speeds of the solution to a Riemann problem with initial data $U^{-}$and $U^{+}$can also be obtained from the fundamental diagram in the $(\rho, \rho v)-$ plane. Hence, we can equivalently discuss a Riemann problem for the first-order LWR model $\partial_{t} \eta+\partial_{x}(\eta v(\eta))=0$ with left Riemann data given by $\eta^{-}$for $\eta^{-}=\rho_{1}^{-}+\rho_{2}^{-}$. Then, the assertion of the proposition is immediate, see e.g. [16, 15].

Similarly, we obtain a result for admissible states for an outgoing road $k \in \delta_{n}^{+}$. In this case the initial data $U^{+}$for $(3.5)$ is given $\left(U^{+}=U_{k, 0}\right)$ and we determine all states $U^{-}$, such that the solution $U(x, t)$ to $(3.5)$ is either a constant or contains waves of positive speed, only. Again, we call such states $U^{-}$admissible for the outgoing road and exclude stationary shocks and the vacuum from the discussion.

Proposition 3.2. Consider an admissible initial data $U^{+}=\left(\rho_{1}^{+}, \rho_{2}^{+}\right)$and an outgoing road $k$. Let $q \in \mathbb{R}$ be an arbitrary given flux, such that

$$
0<q \leq s\left(\rho^{+}\right)
$$


Then, a state $U^{-}=\left(\rho_{1}^{-}, \rho_{2}^{-}\right)$is admissible for the outgoing road, iff $\rho_{1}^{-}+\rho_{2}^{-}=\eta^{-}$. Herein, $\eta^{-}$is the unique value $\eta^{-} \in(0,1]$, such that $q=\eta^{-} v\left(\eta^{-}\right)$and such that the solution to the Riemann problem for the scalar LWR model,

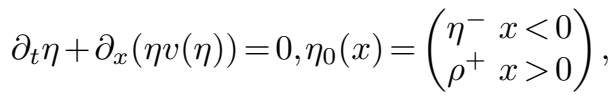

consists of waves of non-negative speed.

Proof. The proof is slightly more involved than in the previous case, since a solution $U(x, t)$ to $(3.5)$ is in general a composition of waves of the first and second family. First note, that as in $[16,15]$, we obtain a unique value $\eta^{-}$with the properties stated above. Now, assume we have a Riemann datum $U^{-}=\left(\rho_{1}^{-}, \rho_{2}^{-}\right)$which satisfies $\eta^{-}=\rho_{1}^{-}+\rho_{2}^{-}$. As in the previous paragraph the Riemann problem (3.5) admits a solution $U(x, t)$ with possible intermediate state $U^{*}=\rho^{+} / \rho^{-}\left(\begin{array}{c}\rho_{1}^{-} \\ \rho_{2}^{-}\end{array}\right)$. Obviously, the 2-wave connecting $U^{*}$ and $U^{+}$has non-negative speed. Moreover, as in the proof of the previous proposition, the speed of the 1-wave connecting $U^{-}$to $U^{*}$ is given by the wave speed of the solution to a Riemann problem for the scalar LWR equation (3.10) with initial data $\left(\rho^{-}, \rho^{*}\right)$. Now, $\rho^{-}=\eta^{-}$and due to proposition $2.3, \rho^{*}=\rho^{+}$. Hence, $s\left(\rho^{*}\right)=s\left(\rho^{+}\right)$and by construction of $\eta^{-}$, the solution to (3.10) consists of waves of non-negative speed.

In proposition 3.2, there is no uniqueness for the admissible states $U^{-}$given, only its total density $\rho_{1}^{-}+\rho_{2}^{-}$is uniquely given by $\eta^{-}$. Again, propositions 3.1 and 3.2 show the fact, that the proposed model is a reformulation of the LWR model combined with an additional advection equation. However, in the case of road intersections the proposed reformulation proves useful, cf. the following discussion.

3.2. The 1-1 junction. We consider a network consisting of just one junction with degree 2 and construct a weak solution in the sense of (3.3) for constant initial data $U_{0}^{i}, i=1,2$. Note, that the different arcs $i=1,2$ might have different free flow velocities $c^{i}$, cf. (3.2), due to changed road conditions. Let $x_{n}^{1}=x_{n}^{2}$ and recall $\rho^{i}=$ $\rho_{1}^{i}+\rho_{2}^{i}$.

Proposition 3.3. Let $U_{0}^{i}, i=1,2$ be some given admissible initial data, constant on each arc $i=1,2$. Then there exists a unique weak solution $\left\{U^{1}=\left(\rho_{1}^{1}, \rho_{2}^{1}\right), U^{2}=\left(\rho_{1}^{2}, \rho_{2}^{2}\right)\right\}$ in the sense of (3.3) with the following properties:

1. Both moments are conserved through the junction, i.e., (3.4) holds for $t>0$.

2. The total flux $\rho^{1} v^{1}\left(\rho^{1}\right)=\rho^{2} v^{2}\left(\rho^{2}\right)$ is maximal at the interface $(x, t)=$ $\left(x_{n}^{1}, t\right), t>0$.

Proof. We define $U^{1-}:=U_{0}^{1}$ and $U^{2+}:=U_{0}^{2}$ and consider the following maximization problem for the unknown $\rho_{1}^{1+}, \rho_{2}^{1+}, \rho_{1}^{2-}$ and $\rho_{2}^{2-}$ :

$$
\begin{aligned}
& \max \rho^{1+} v^{1}\left(\rho^{1+}\right) \text { subject to } \\
& \rho_{1}^{1+} v^{1}\left(\rho^{1+}\right)=\rho_{1}^{2-} v^{2}\left(\rho^{2-}\right), \\
& \rho_{2}^{1+} v^{1}\left(\rho^{1+}\right)=\rho_{2}^{2-} v^{2}\left(\rho^{2-}\right), \\
& 0<\rho^{1+} v^{1}\left(\rho^{1+}\right) \leq d\left(\rho^{1-}\right) \\
& 0<\rho^{2-} v^{2}\left(\rho^{2-}\right) \leq s\left(\rho^{2+}\right) .
\end{aligned}
$$


Here, $s=s^{2}$ and $d=d^{1}$ denote the supply and demand function for the outgoing and incoming road, respectively. Introducing $q^{1}:=\rho^{1+} v^{1}\left(\rho^{1+}\right)$ we obtain $q^{1}=\min \left\{d\left(\rho^{1-}\right), s\left(\rho^{2+}\right)\right\}$ to be the maximal total flux at the interface. Due to Proposition 3.1 we obtain a unique state $U^{1+}=\left(\rho_{1}^{1+}, \rho_{2}^{1+}\right)$, such that $\rho^{1+} v^{1}\left(\rho^{1+}\right)=q^{1}$. Due to proposition 3.2 we obtain a unique total density $\left(\eta^{-}=\right) \rho^{2-}$ such that $\rho^{2-} v^{2}\left(\rho^{-}\right)=q^{1}$. Moreover, (3.11b) and (3.11c) then uniquely determine $\rho_{1}^{2-}$ and $\rho_{2}^{2-}$, since $v^{2}\left(\rho^{2-}\right)>0$ by (3.11e). Finally, $U^{1}$ is obtained as a solution to the half-Riemann problem (3.5) with initial data $U^{-}:=U^{1-} \equiv U_{0}^{1}$ and $U^{+}:=U^{1+}$. The solution $U^{2}$ is obtained as a solution to (3.5) with initial data $U^{-}:=U^{2-}$ and $U^{+}:=U^{2+} \equiv U_{0}^{2}$. Due to Proposition 3.1 and $3.2, U^{i}$ is a superposition of waves of the first and (for $i=2$ ) second family having only non-positive (resp. non-negative) wave speeds. We have $U^{1}\left(x_{n}^{1}, t\right)=U^{1+}$ and $U^{2}\left(x_{n}^{2}, t\right)=U^{2-}$ for $t>0$ and therefore, by construction, the assertions of the proposition are fulfilled.

REMARK 3.1. In the case of $c^{i}=c, i=1,2$ the junction is artificial and a weak solution to (3.3) is given by the entropy solution of a standard Riemann problem for (2.9) with initial data $U_{0}=\left(\begin{array}{cc}U_{0}^{1} & x<x_{n}^{1} \\ U_{0}^{2} & x>x_{n}^{2}\end{array}\right)$. This solution is the same as obtained by the previous proposition.

3.3. The 2-1 and 1-2 junction. First, we consider a network consisting of three connected $\operatorname{arcs} i=1,2,3$ at a vertex $n$ with $\left|\delta_{n}^{-}\right|=2$, i.e., a situation where two roads merge into one other road $(i=3)$. As before, we consider the case of constant initial data $U_{0}^{i}, i=1,2,3$ and the following proposition guarantees the existence of a weak solution in the sense of (3.3): In fact, the only relevant change to the previous discussion is the formulation of a suitable maximization problem to obtain the right $(i=1,2)$ and left $(i=3)$ initial data for the half-Riemann problems (3.5). In the current case, we maximize the total incoming flux. We do not obtain a unique solution, since the maximization does not necessarily possess a unique solution. It is possible to introduce additional conditions to obtain uniqueness, as for example below, in the case of a vertex of arbitrary degree. Such conditions are subject to the particular modeling of the road intersection and have been studied for example in [5, 13, 15].

Proposition 3.4. Let $U_{0}^{i}, i=1,2,3$ be some given admissible initial data, constant on each arc $i=1,2,3$. Then, there exists a (not necessarily unique!) weak solution $\left\{U^{1}=\right.$ $\left.\left(\rho_{1}^{1}, \rho_{2}^{1}\right), U^{2}=\left(\rho_{1}^{2}, \rho_{2}^{2}\right), U^{3}=\left(\rho_{1}^{3}, \rho_{2}^{3}\right)\right\}$ in the sense of (3.3) with the following properties:

1. The moments are conserved through the junction, i.e., (3.4) holds for $t>0$.

2. The total flux $\rho^{1} v^{1}\left(\rho^{1}\right)+\rho^{2} v^{2}\left(\rho^{2}\right)$ is maximal at the interface $(x, t)=$ $\left(x_{n}^{1}, t\right), t>0$.

Proof. The proof is analogous to the proof of Proposition 3.3, but instead of (3.11), we consider the following problem for the unknowns $U^{1+}, U^{2+}, U^{3-}$ and with given data $U^{1-}=U_{1}^{0}, U^{2-}=U_{2}^{0}$ and $U^{3+}=U_{3}^{0}$,

$$
\begin{aligned}
& \max \rho^{2+} v^{2}\left(\rho^{2+}\right)+\rho^{1+} v^{1}\left(\rho^{1+}\right) \text { subject to } \\
& \rho_{1}^{1+} v^{1}\left(\rho^{1+}\right)+\rho_{1}^{2+} v^{2}\left(\rho^{2+}\right)=\rho_{1}^{3-} v^{3}\left(\rho^{3-}\right), \\
& \rho_{2}^{1+} v^{1}\left(\rho^{1+}\right)+\rho_{2}^{2+} v^{2}\left(\rho^{2+}\right)=\rho_{2}^{3-} v^{3}\left(\rho^{3-}\right), \\
& 0<\rho^{1+} v^{1}\left(\rho^{1+}\right) \leq d_{1}\left(\rho^{1-}\right), \\
& 0<\rho^{2+} v^{2}\left(\rho^{2+}\right) \leq d_{2}\left(\rho^{2-}\right), \\
& 0<\rho^{3-} v^{3}\left(\rho^{3-}\right) \leq s_{3}\left(\rho^{3+}\right) .
\end{aligned}
$$


Assume that $s_{3}\left(\rho^{3+}\right) \geq d_{1}\left(\rho^{1-}\right)+d_{2}\left(\rho^{2-}\right)$, then, as in the previous proof, the problem $(3.12)$ has a unique solution. We refer to $[13,5,15]$ for additional constraints guaranteeing uniqueness in the case $s_{3}\left(\rho^{3+}\right) \leq d_{1}\left(\rho^{1-}\right)+d_{2}\left(\rho^{2-}\right)$. For any solution to (3.12) we proceed as before and obtain a weak solution to (3.3) as a solution to the three half-Riemann problems (3.5) with initial data $U^{i-}$ and $U^{i+}$ for $i=1,2,3$, respectively.

REMARK 3.2. In [10] the existence of a unique weak solution at an arbitrary junction has been proven. This is achieved by introducing additional constraints and a modified objective function to maximize. We propose a different choice below for a vertex of arbitrary degree, i.e., an "equal priority rule" as in [15].

Before discussing the general case, we consider a vertex of degree three with two outgoing roads $i=2,3$. For this case we propose the following modeling: We introduce a distribution rate $\alpha \in[0,1]$ of the total incoming flux $\rho\left(x_{n}^{1}, t\right) v^{1}\left(\rho\left(x_{n}^{1}, t\right)\right)$. To be more precise, we prove existence of a weak solution $\left\{U^{i}\right\}_{i=1}^{3}$ which satisfies

$$
\begin{aligned}
\alpha \rho_{1}^{1} v^{1}\left(\rho^{1}\right)\left(x_{n}^{1}, t\right) & =\rho_{1}^{2} v^{2}\left(\rho^{2}\right)\left(x_{n}^{2}, t\right), \\
\alpha \rho_{2}^{1} v^{1}\left(\rho^{1}\right)\left(x_{n}^{1}, t\right) & =\rho_{2}^{2} v^{2}\left(\rho^{2}\right)\left(x_{n}^{2}, t\right), \\
(1-\alpha) \rho_{1}^{1} v^{1}\left(\rho^{1}\right)\left(x_{n}^{1}, t\right) & =\rho_{1}^{3} v^{3}\left(\rho^{3}\right)\left(x_{n}^{3}, t\right), \\
(1-\alpha) \rho_{2}^{1} v^{1}\left(\rho^{1}\right)\left(x_{n}^{1}, t\right) & =\rho_{2}^{3} v^{3}\left(\rho^{3}\right)\left(x_{n}^{3}, t\right) .
\end{aligned}
$$

Obviously, (3.13) implies (3.4) and we have a distribution as $\alpha \rho^{1} v^{1}\left(\rho^{1}\right)=\rho^{2} v^{2}\left(\rho^{2}\right)$. Under the additional assumption (3.13), the following result holds true.

Proposition 3.5. Let $U_{0}^{i}, i=1,2,3$ be some given admissible initial data, constant on each arc $i=1,2,3$, and a distribution rate $0 \leq \alpha \leq 1$. Then, there exists a unique weak solution $\left\{U^{1}=\left(\rho_{1}^{1}, \rho_{2}^{1}\right), U^{2}=\left(\rho_{1}^{2}, \rho_{2}^{2}\right), U^{3}=\left(\rho_{1}^{3}, \rho_{2}^{3}\right)\right\}$ in the sense of (3.3) with the following properties:

1. The moments are conserved through the junction, i.e., (3.4) holds for $t>0$, and they are distributed according to $\alpha$, i.e., (3.13) holds.

2. The total flux $\rho^{1} v^{1}\left(\rho^{1}\right)$ is maximal at the interface $(x, t)=\left(x_{n}^{1}, t\right), t>0$.

The proof is similar to the one of the two previous propositions and is therefore omitted. For later reference, we remark that the maximal flux at the interface $x=x_{n}^{i}$ for any time $t>0$ in the non-trivial case $\alpha \in(0,1)$, is given by

$$
q(t)=q=\min \left\{d_{1}\left(\rho^{1}\right), s_{2}\left(\rho^{2}\right) / \alpha, s_{3}\left(\rho^{3}\right) /(1-\alpha)\right\},
$$

where $\rho^{i}=\rho_{1,0}^{i}+\rho_{2,0}^{i}$ is given by the initial data.

REMARK 3.3. The following extension to the single distribution rate $\alpha$ is possible: We introduce two rates $\alpha^{1}$ and $\alpha^{2}$, both in [0,1], and distribute the partial incoming fluxes $\rho_{1}^{1} v^{1}\left(\rho^{1}\right)$ and $\rho_{2}^{1} v^{1}\left(\rho^{1}\right)$ with possibly different rates $\alpha^{1}$ and $\alpha^{2}$, i.e., we replace the equations in (3.13) by

$$
\begin{aligned}
\alpha^{1} \rho_{1}^{1} v^{1}\left(\rho^{1}\right)\left(x_{n}^{1}, t\right) & =\rho_{1}^{2} v^{2}\left(\rho^{2}\right)\left(x_{n}^{2}, t\right), \\
\alpha^{2} \rho_{1}^{1} v^{1}\left(\rho^{1}\right)\left(x_{n}^{1}, t\right) & =\rho_{2}^{2} v^{2}\left(\rho^{2}\right)\left(x_{n}^{2}, t\right), \\
\left(1-\alpha^{1}\right) \rho_{1}^{1} v^{1}\left(\rho^{1}\right)\left(x_{n}^{1}, t\right) & =\rho_{1}^{3} v^{3}\left(\rho^{3}\right)\left(x_{n}^{3}, t\right), \\
\left(1-\alpha^{2}\right) \rho_{1}^{1} v^{1}\left(\rho^{1}\right)\left(x_{n}^{1}, t\right) & =\rho_{2}^{3} v^{3}\left(\rho^{3}\right)\left(x_{n}^{3}, t\right) .
\end{aligned}
$$


But, then the maximization problem for the interface flux $q$ does not necessarily pose a unique solution.

3.4. The general $\mathbf{m}-\mathbf{n}$ junction. Combining the discussion of the $1-$ 2 and 2-1 junction, we now state the result for a network with a single vertex with $i \in\{1, \ldots, m\}=\delta_{n}^{-}$incoming and $i \in\{m+1, \ldots, n+m\}=\delta_{n}^{+}$outgoing roads. We need to introduce additional conditions to obtain a unique solution $\left\{U^{i}\right\}_{i=1}^{n+m}$ in the sense of (3.3). First and as in [10], we introduce a distribution matrix $A=\left(\alpha_{i j}\right)_{i j} \in \operatorname{Mat}\left(\mathbb{R}^{m}, \mathbb{R}^{n}\right)$ such that

$$
\sum_{j \in \delta_{n}^{+}} \alpha_{i j}=1, \forall i \in \delta_{n}^{-}
$$

Second, we introduce an equal priority rule as in [15]: If cars (of either class) coming from more than one incoming road move towards the same outgoing road, we assume that they enter this road turn by turn. In particular, we model this fact by looking for weak solutions which additionally satisfy $\rho^{i} v^{i}\left(\rho^{i}\right)=\rho^{k} v^{k}\left(\rho^{k}\right)$ for all $i, k \in \delta_{n}^{-}$.

Summarizing, we have the following theorem on existence and uniqueness of a weak solution $\{U\}_{i=1}^{n+m}$ to a piecewise constant initial data.

THEOREM 3.1. Consider admissible initial data $U_{0}^{i}$ constant on each arc $i=1, \ldots, n+$ $m=\delta_{n}^{-} \cup \delta_{n}^{+}$and a flux distribution matrix $A \in M a t\left(\mathbb{R}^{m}, \mathbb{R}^{n}\right)$ satisfying (3.15) and such that $\sum_{i} \alpha_{i j} \neq 0$.

Then, there exists a unique weak solution $\left\{U^{i}\right\}$ to (3.3) which satisfies both (3.4) and the following additional properties:

1. The moments are distributed according to A, i.e.,

$$
\sum_{i \in \delta_{n}^{-}} \alpha_{i j} \rho_{l}^{i} v^{i}\left(\rho^{i}\right)\left(x_{n}^{i}, t\right)=\rho_{l}^{j} v^{j}\left(\rho^{j}\right)\left(x_{n}^{j}, t\right), \quad l=1,2, j \in \delta_{n}^{+}, t>0
$$

2. An equal priority rule holds true, i.e.,

$$
\rho^{i} v^{i}\left(\rho^{i}\right)\left(x_{n}^{i}, t\right)=\rho^{j} v^{j}\left(\rho^{j}\right)\left(x_{n}^{j}, t\right), i, j \in \delta_{n}^{-}, t>0
$$

3. The total incoming flux $\sum_{i \in \delta_{n}^{-}} \rho^{i} v^{i}\left(\rho^{i}\right)(x, t)$ is maximal at the interface $x=$ $x_{n}^{i}, t>0$.

Thanks to (3.15) we obtain (3.4) from (3.16). The restriction $0<\sum_{i} \alpha_{i j}$ has been imposed to avoid triviality due to an used outgoing road.

Proof. (of Theorem 3.1) We introduce $U^{i-}=U_{0}^{i}, i \in \delta_{n}^{-}$and $U^{j+}=U_{0}^{j}, j \in \delta_{n}^{+}$. To prove the assertion we consider the following maximization problem in the unknowns $\left(U^{i+}, U^{j-}\right)_{i, j}$ for $i \in \delta_{n}^{-}$and $j \in \delta_{n}^{+}$. 


$$
\begin{aligned}
& \max \sum_{i \in \delta_{n}^{-}} \rho^{i+} v^{i}\left(\rho^{i+}\right) \text { subject to } \\
& \sum_{i \in \delta_{n}^{-}} \alpha_{i j} \rho_{l}^{i+} v^{i}\left(\rho^{i+}\right)=\rho_{l}^{j-} v^{j}\left(\rho^{j-}\right), l=1,2, j \in \delta_{n}^{+}, \\
& 0<\rho^{i+} v^{i}\left(\rho^{i+}\right) \leq d_{i}\left(\rho^{i-}\right), i \in \delta_{n}^{-}, \\
& 0<\rho^{j-} v^{j}\left(\rho^{j-}\right) \leq s_{j}\left(\rho^{j+}\right), j \in \delta_{n}^{+}, \\
& \rho^{i+} v^{i}\left(\rho^{i+}\right)=\rho^{k+} v^{k}\left(\rho^{k+}\right), i, k \in \delta_{n}^{-} .
\end{aligned}
$$

If we introduce $\tilde{q}:=\rho^{i+} v^{i}\left(\rho^{i+}\right)=\rho^{k+} v^{k}\left(\rho^{k+}\right), i, k \in \delta_{n}^{-}$, we obtain that $\tilde{q} \in \mathbb{R}$ is uniquely determined as

$$
\tilde{q}=\min \left\{d_{i}\left(\rho^{i-}\right), s_{j}\left(\rho^{j+}\right) / \sum_{i} \alpha_{i j}: i \in \delta_{n}^{-}, j \in \delta_{n}^{+}\right\}
$$

Due to proposition 3.1 we obtain a unique state $U^{i+}=\left(\rho_{1}^{i+}, \rho_{2}^{i+}\right)$ for every incoming road $i \in \delta_{n}^{+}$, such that $\rho^{i+} v^{i}\left(\rho^{i+}\right)=\tilde{q}$. Moreover, $U^{i}(x, t)$ is given as solution to the half-Riemann problem (3.5) with initial data $U^{i-}$ and $U^{i+}$ and consists of waves having non-positive wave speed. Then, due to $(3.18 \mathrm{~b})$ and proposition 3.2 we obtain a unique state $U^{j-}=\left(\rho_{1}^{j-}, \rho_{2}^{j-}\right)$ for each outgoing road $j \in \delta_{n}^{+}$and such that the solution $U^{j}$ to the half-Riemann problem (3.5) with initial data $U^{j-}$ and $U^{j+}$ is a superposition of waves of non-negative speed. Finally, $\left\{U^{i}\right\}$ fulfills (3.16) and (3.17) by construction.

\section{Numerical results}

All the following results have been obtained by a second-order relaxed scheme, see for example [18]. At the intersection the states are determined as in the previous section. The results presented are obtained with a discretization of $\Delta x=1 / 800$.
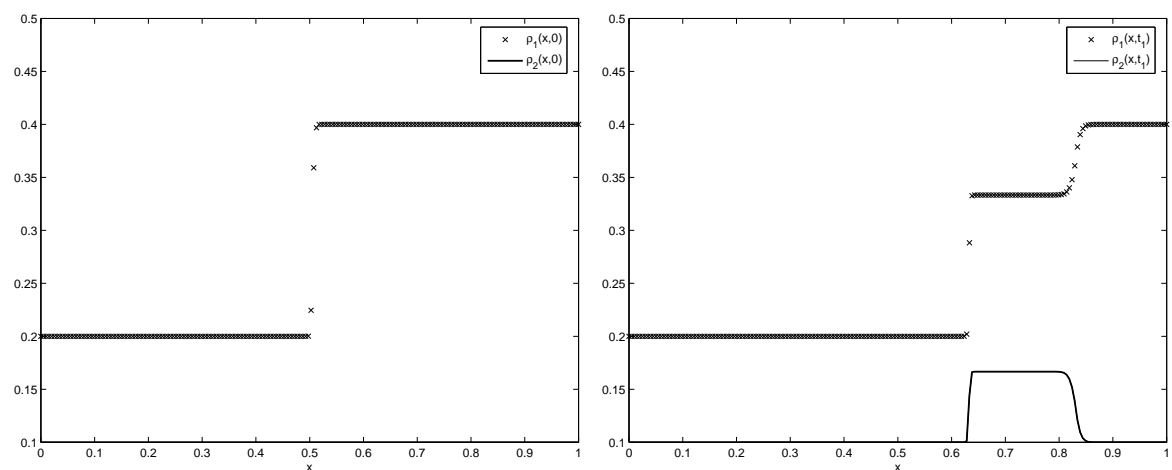

FIG. 4.1. Densities $\rho_{1}$ and $\rho_{2}$ for two connected roads with equal free flow velocities at time $t=0$ and $t=1$

First, we consider a situation of a 1-1 intersection. We have to two connected roads $i=1,2$ with possibly different free flow velocities $c^{i}$, cf. equation (3.2). We 

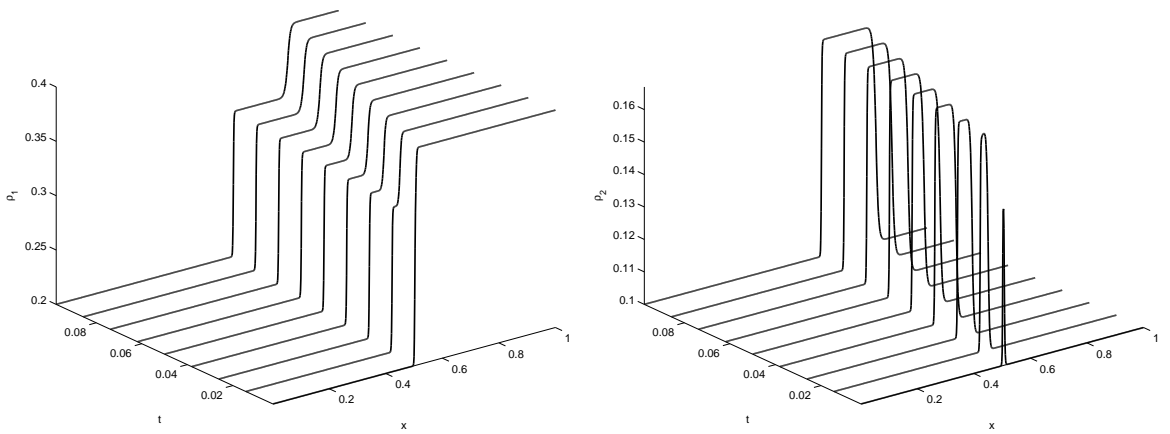

FIG. 4.2. Densities $\rho_{1}$ and $\rho_{2}$ for two connected roads with equal free flow velocities.
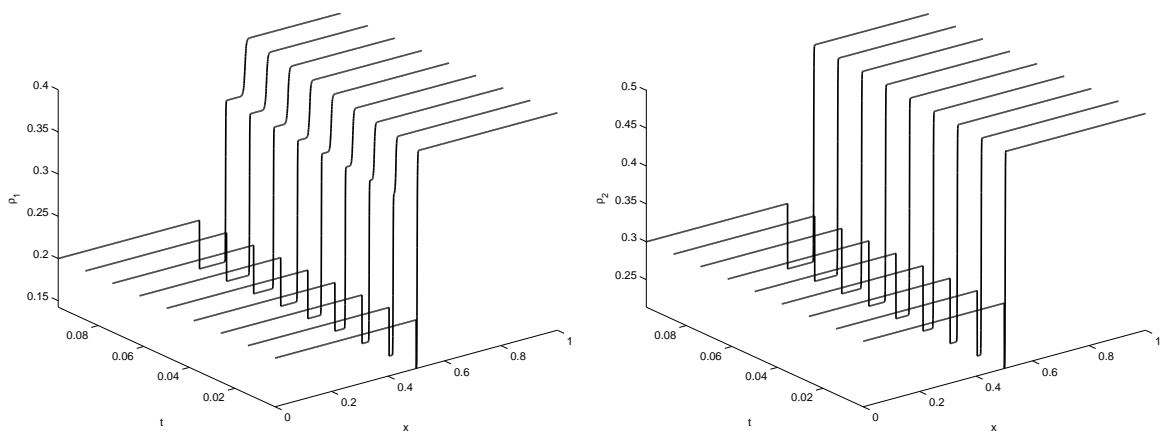

FIG. 4.3. Densities $\rho_{1}$ and $\rho_{2}$ for two connected roads with different free flow velocities

prescribe initial data $U_{0}^{i}$ to show the time-evolution of the solution $U^{i}(x, t)$ obtained in Section 3.2.

The details are as follows. The intersection is located at $x_{n}^{1}=x_{n}^{2}=1 / 2$ and the initial data is

$$
U_{0}^{1}=\left(\begin{array}{l}
0.2 \\
0.1
\end{array}\right), \quad U_{0}^{2}=\left(\begin{array}{l}
0.4 \\
0.1
\end{array}\right) .
$$

In the case of the same free flow velocity

$$
c^{1}=c^{2}=1,
$$

the numerical solution is given in Figure 4.2. The Riemann data chosen is such that there is a discontinuity of the total density $\rho_{0}^{1}+\rho_{0}^{2}$ at time $t=0$ and $x=1 / 2$. We then observe that this discontinuity travels with positive speed and we show the distribution of this density among the two classes $\rho_{1}$ and $\rho_{2}$ at time $t=0$ and $t=1$ in Figure 4.1. The time-evolution of both solutions $\rho_{1}(x, t)$ and $\rho_{2}(x, t)$ is presented in Figure 4.2. As asserted in the previous section, the solution for each class $\rho_{i}$ consists at most of three connected states which can be nicely seen in the picture. This serves as a benchmark for the numerical code and shows in particular, that the code resolves the arising contact discontinuities.

Second, we consider a similar problem as before but for different free flow velocities on the connected roads. This can be seen as solving a conservation law with 
discontinuous coefficient. The chosen velocities are

$$
c^{1}=0.8 \text { and } c^{2}=1,
$$

and the initial data is given by

$$
U_{0}^{1}=\left(\begin{array}{c}
0.2 \\
0.1
\end{array}\right), \quad U_{0}^{2}=\left(\begin{array}{c}
0.3 \\
0.5
\end{array}\right)
$$

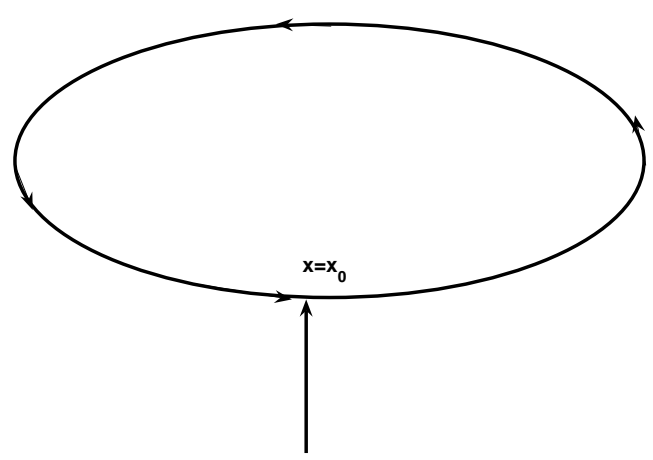

Fig. 4.4. Sketch of the ring road.

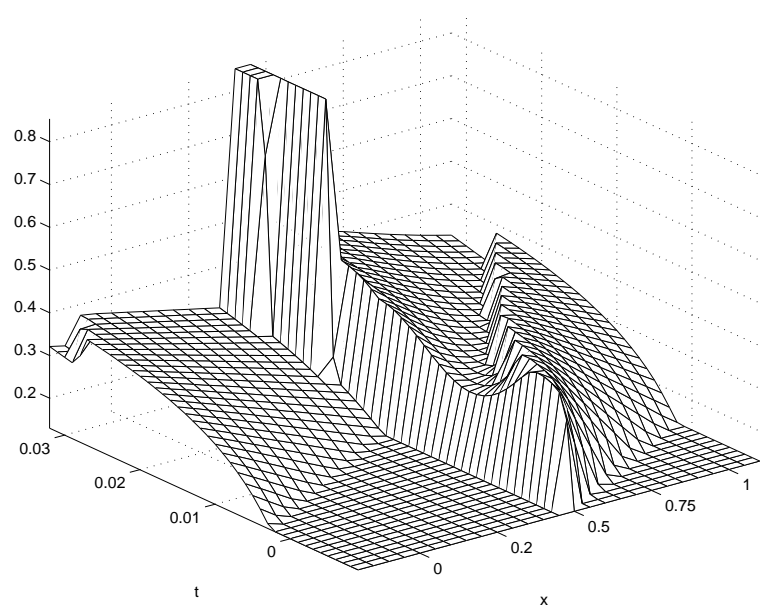

FIG. 4.5. Total density $\rho(x, t)$ on the ring road $i=1$.

In Figure 4.3 we present the time-evolution of the densities $\rho_{1}^{i}$ and $\rho_{2}^{i}$ on both roads $i=1,2$. Due to the low inflow $\rho^{1} v^{1}\left(\rho^{1}\right)$, the constant state $U_{0}^{1}$ is preserved on the incoming road $i=1$. On the outgoing road $i=2$, we observe a composition of a $1-$ and a 2 -wave. Neither $\rho_{1}$ nor $\rho_{2}$ has to be continuous through the intersection, but (as required by the coupling conditions) this holds true for the flux $q^{i}$ (not shown). 

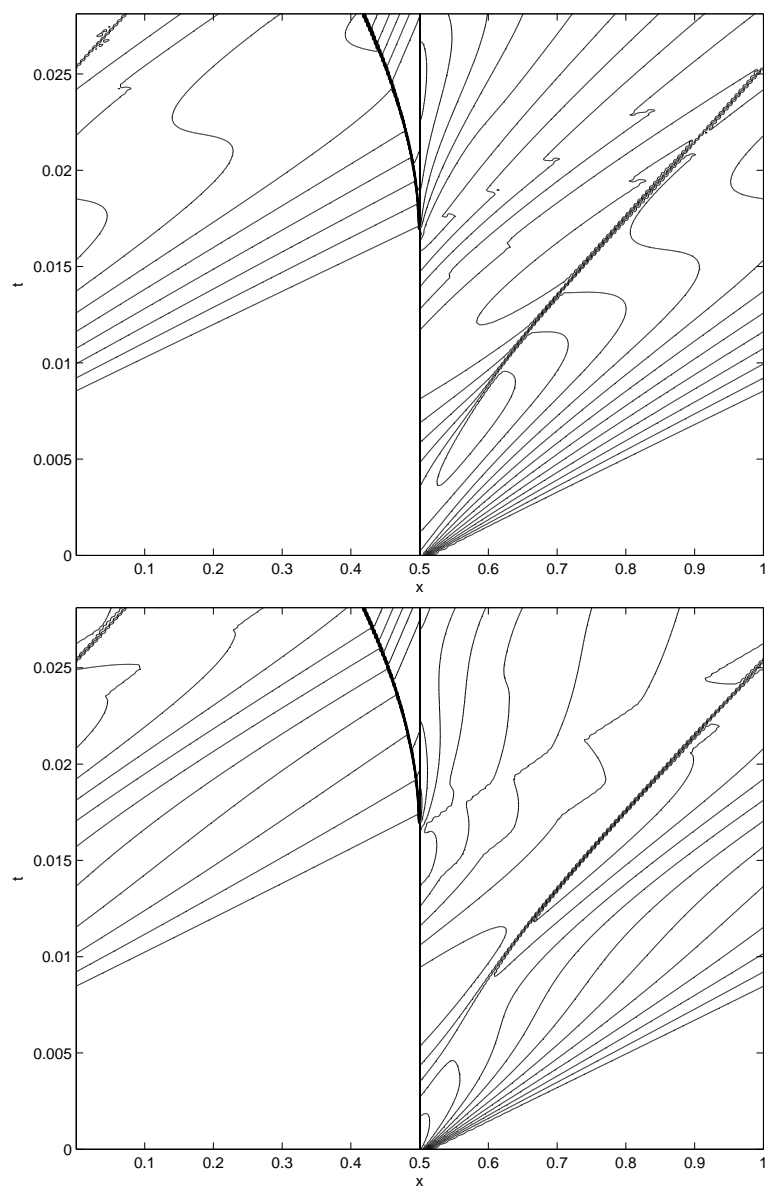

FIG. 4.6. Densities $\rho_{1}(x, t)$ (top) and $\rho_{2}(x, t)$ (bottom) on the ring road $i=1$.

Next, we present results on a ring road represented in Figure 4.4, i.e., with two roads $i=1,2$.

This ring road has an inflow arc $i=2$ at $x=x_{0}$ where we prescribe a time-varying inflow in both the $\rho_{1}$ and $\rho_{2}$. At first this generates waves on the part of the road $x>x_{0}$. These waves now travel along the road and reach after some time $(\Delta t \approx 0.0152)$ again the intersection at $x=x_{0}$. Now, the combined inflow of the circular road $(i=1)$ and the additional road, exceeds the maximal possible flow. Hence, we observe a shock wave forming and moving on $x<x_{0}$. This pattern can be seen in all subsequent pictures. Moreover, we apply a slightly different condition at the intersection $x=$ $x_{0}$ : Instead of requiring an equal flux condition (as in theorem 3.1) we propose the following: If the sum of the demands $d_{1}\left(\rho^{1}\left(x_{0}+, t\right)\right)+d\left(\rho^{2}\left(x_{0}, t\right)\right)$ does not exceed the supply $s_{3}\left(\rho^{1}\left(x_{0}+, t\right)\right)$, then we allow all the flow to pass the intersection. Otherwise, we impose (3.17). This condition is as in [13] and also allows for a unique solution of $(3.18)$, see $[13,5]$.

We depict the numerical results for the circular road only. In this case, the drawing area is such that $x=0$ and $x=1$ correspond to the same point in the network graph. The intersection is located at $x=x_{0}=1 / 2$ and we set $c=1$ for all roads. Along 
$\left(x_{0}, t\right), t<0.0152$, we see the influence of inflow of road $i=2$ leading to increasing and decreasing total flux. Further, we observe the backwards moving shock wave after $\Delta t=0.0152$. Additionally, we plot the density $\rho(x, t)$ in Figure 4.5 where the shock wave can be seen also. For completeness, we also give the results on contour plots of the densities $\rho_{1}$ and $\rho_{2}$ on road $i=1$ in Figure 4.6.

Summary. We extended a multi-class traffic flow model with two different classes to a network. We proposed coupling conditions by analyzing Riemann problems at the intersection. We presented some numerical validations of these conditions on a 1-1 intersection with possibly different free flow velocities and on a ring road having only one inflow arc. In the latter example the numerical results show that the coupled dynamics of the two class model yields a complex shock and rarefaction wave pattern. Further numerical examples will be considered in a forthcoming publication. Moreover, we are currently working on the extension of the results to Cauchy initial data. In the same spirit as in [10], this might be achieved by careful TV estimates on the wave interactions at intersections.

Acknowledgement. This work has been partially supported by the Kaiserslautern Excellence Cluster "Dependable Adaptive Systems and Mathematical Modeling" and "Graduiertenkolleg Mathematik und Praxis" of the University of Kaiserslautern and by the French ACI-NIM (Nouvelles Interactions des Mathématiques) $N^{o}$ 193 (2004).

\section{REFERENCES}

[1] A. Aw, A. Klar, M. Materne and M. Rascle, Derivation of continuum flow traffic models from microscopic follow the leader models, SIAM J. Appl. Math., 63, 259-289, 2002.

[2] A. Aw and M. Rascle, Resurrection of second order models of traffic flow? SIAM J. Appl. Math., 60, 916-944, 2000.

[3] P. Bagnerini and M. Rascle, A multi-class homogenized hyperbolic model of traffic flow, SIAM J. Math. Anal., (4) 35, 949-973, 2003.

[4] S. Benzoni-Gavage and R. M. Colombo, An n-population model for traffic flow, European J. Appl. Math., 63, 818-833, 2003.

[5] G. M. Coclite, M. Garavello and B. Piccoli, Traffic flow on road networks, SIAM J. Math. Anal., 36, 1862-1886, 2005.

[6] C. M. Dafermos, Hyperbolic Conservation Laws in Continuum Physics, Springer Verlag, Berlin, Heidelberg, New York, 2000.

[7] C. F. Daganzo, A behavioral theory of multi-lane traffic flow part I: Long homogeneous freeway sections, Trans. Res. B, 36, 131-158, 2002.

[8] C. F. Daganzo, A behavioral theory of multi-lane traffic flow part II: Merges and the onset of congestion, Trans. Res. B, 36, 159-169, 2002.

[9] M. Garavello and B. Piccoli, Traffic flow on a road network using the Aw-Rascle model, Comm. Partial Differential Equations, to appear.

[10] M. Garavello and B. Piccoli, Source-Destination flow on a road network, Comm. Math. Sci., 3, 261-283, 2005.

[11] J. Greenberg, Extension and amplification of the Aw-Rascle model, SIAM J. Appl. Math., 62, 729-745, 2001.

[12] J. Greenberg, A. Klar and M. Rascle, Congestion on multilane highways, SIAM J. Appl. Math., 63, 818-833, 2003.

[13] M. Herty and A. Klar, Modelling, simulation and optimization of traffic networks, SIAM J. Sci. Comp., 25, 1066-1087, 2004.

[14] M. Herty, S. Moutari and M. Rascle, Optimization criteria for modeling intersections of vehicular traffic flow, Networks and Heterogenous Media, 1, 275-294, 2006.

[15] M. Herty and M. Rascle, Coupling conditions for a class of second-order models for traffic flow, SIAM J. Math. Anal., to appear, 2006.

[16] H. Holden and N. H. Risebro, A mathematical model of traffic flow on a network of unidirectional roads, SIAM J. Math. Anal., 26, 999-1017, 1995. 
[17] R. Illner, A. Klar and M. Materne, Vlasov-Fokker-Planck models for multilane traffic flow, Comm. Math. Sci., 1, 1-12, 2003

[18] S. Jin and Z. Xin, The relaxation schemes for systems of conservation laws in arbitrary space dimensions, Comm. Pure Appl. Math., 48, 235-255, 1995.

[19] A. Klar and R. Wegener, Kinetic derivation of macroscopic anticipation models for vehicular traffic, SIAM J. Appl. Math., 60, 1749-1766, 2000.

[20] N. S. Kruzkov, First order quasi linear equations in several independent variables, Math. USSR Sbornik, 10, 217-243, 1970.

[21] J. P. Lebacque, Les modèles macroscopiques du traffic, Annales des Ponts et Chaussées, 63, 24-45, 1993.

[22] J. P. Lebacque and M. Khoshyaran, First order macroscopic traffic flow models: Intersection modeling, network modeling, Proceedings of the 16th International Symposium on Transportation and Traffic Theory (ISTTT), Elsevier, 365-386, 2005.

[23] M. J. Lighthill and G. B Whitham, On kinematic waves, Proc. Royal Soc. Edinburgh, 229, 281-316, 1955.

[24] H. J. Payne, FREFLO: A macroscopic simulation model for freeway traffic, Transportation Research Record, 722, 68-77, 1979.

[25] P. I. Richards, Shock waves on the highway, Operation Research, 4, 42-51, 1956.

[26] G. C. K. Wong and S. C. Wong, A multi-class traffic flow model - an extension of the LWR model with heterogeneous drivers, Transp. Research A, 36, 827-841, 2002.

[27] P. Zhang, R. Liu, S. C. Wong and S. Dai, Hyperbolicity and kinematic waves of a class of multi-population partial differential equations, European J. Appl. Math., to appear.

[28] M. Zhang, C.-W. Shu, G. C. K Wong and S. C. Wong, A weighted essentially non-oscillatory numerical scheme for a multi-class Lighthill-Whitham-Richards traffic flow model, J. Comp. Phys., 191, 639-659, 2003. 